\title{
ANNIHILATORS OF MODULES WITH A FINITE FREE RESOLUTION
}

\author{
WOLMER V. VASCONCELOS ${ }^{1}$
}

Abstract. Let $A$ be a commutative ring and let $E$ be an $A$ module with a finite free resolution (see below for definition). Extending results known previously for noetherian rings, it is shown that $\operatorname{ann}(E)=$ annihilator of $E$ is trivial if and only if the Euler characteristic of $E=\chi(E)>0$; in addition, if $\chi(E)=0, \operatorname{ann}(E)$ is dense (i.e. $\operatorname{ann}(\operatorname{ann}(E))=0$ ). Also, a local ring is constructed with its maximal ideal with a finite free resolution but consisting exclusively of zero-divisors and thus, contrary to the noetherian case, one does not always have a nonzero divisor in $\operatorname{ann}(E)$ if $\chi(E)=0$. Finally, if $E$ has a finite resolution by (f.g.) projective modules it turns out that ann $(\operatorname{ann}(E))$ is generated by an idempotent element.

1. For a commutative ring $A$, an $A$-module $E$ has, we recall, a finite free resolution if there is an exact sequence

$$
0 \rightarrow F_{n} \rightarrow \cdots \rightarrow F_{1} \stackrel{\alpha}{\rightarrow} F_{0} \rightarrow E \rightarrow 0
$$

where each $F_{i}$ is $A$-free with a basis of cardinality $r k\left(F_{i}\right)<\infty$. The integer $\chi(E)=\sum_{i=0}^{n}(-1)^{i} r k\left(F_{i}\right)$ is independent of the resolution (see [2], which we shall use as reference) and it is called the Euler characteristic of $E$.

It has been shown that the positivity of $\chi(E)$ is closely related to the faithfulness of $E$ as an $A$-module. In fact, Auslander and Buchsbaum [1, Proposition 6.2] proved that if $A$ is noetherian:

(i) If $\chi(E)>0$ then annihilator of $E=\operatorname{ann}(E)=(0)$.

(ii) If $\chi(E)=0$ then ann $(E)$ contains a nonzero divisor.

Kaplansky in [2, p. 141] asks whether the chain conditions are needed at all. The purpose of this note is to show that the techniques there suffice to prove that in general we have:

Theorem. (a) If $\chi(E)>0$ then $\operatorname{ann}(E)=(0)$.

(b) If $\chi(E)=0$ then ann $(\operatorname{ann}(E))=(0)$.

Received by the editors November 6, 1970.

AMS 1970 subject classifications. Primary 13C10; Secondary 13D05.

Key words and phrases. Annihilator, finite free resolution, Euler characteristic, Fitting invariant.

${ }^{1}$ Partially supported by a Rutgers University Faculty Fellowship and NSF Grant GP-19995. 
An example of a local ring will show that (b) is best possible, i.e. that $\operatorname{ann}(E)$ does not contain always a nonzero divisor.

2. For the proof we recall some points from [2, p. 139]:

(i) If $S$ is a multiplicative set in $A$, then $\chi\left(E_{S}\right)=\chi(E)$.

(ii) Let $A$ be a local ring in which every finitely generated ideal has a nontrivial annihilator; then any $A$-module with a finite free resolution is actually free. In this case, for brevity, we shall say that $A$ is a 0 -ring.

(a) Assume $\chi(E)>0$ and $0 \neq a \in \operatorname{ann}(E)$. Let $J=\operatorname{ann}(a)$ and let $P$ be a prime ideal minimal over $J$. Then, localizing at $P$ we get that $J_{P} \neq A_{P}$ is the annihilator of $a A_{P}$ and so $a_{P} \neq 0$. Also, $P A_{P}$ is minimal over $J_{P}$ and thus every finitely generated ideal $I$ of $A_{P}$ has a power $I^{n} \subset J_{P}$ and so $I^{n} a_{P}=(0)$; it follows easily that $A_{P}$ is a 0 -ring. Thus $E_{P}$ is free of $\operatorname{rank} \chi(E)>0$ and so $\operatorname{ann}\left(E_{P}\right)=(0)=(\operatorname{ann}(E))_{P} \supset a A_{P}$, a contradiction. Hence ann $(E)=(0)$.

(b) Let $F(E)$ be the 0th Fitting invariant of $E$, i.e. if $\alpha$ in $\left(^{*}\right)$ is an $r \times m$ matrix, $F(E)$ is the ideal of $A$ generated by the $m \times m$ minors of $\alpha$. We know that $(\operatorname{ann}(E))^{m} \subset F(E) \subset \operatorname{ann}(E)$. In particular we have $\operatorname{ann}(\operatorname{ann}(E))=(0)$ iff $\operatorname{ann}(F(E))=(0)$. Ann $(F(E))$ is easier to work with since $F(E)$ is finitely generated and so its annihilator "localizes."

Assume then $0 \neq a \in \operatorname{ann}(F(E))$ and write $J=\operatorname{ann}(a)$. Let $P$ be a prime ideal minimal over $J . J_{P} \neq A_{P}$ implies $a_{P} \neq 0$ and $A_{P}$ is a 0-ring. As $\chi\left(E_{P}\right)=\chi(E)=(0), E_{P}=(0)$ and $F(E)_{P}=F\left(E_{P}\right)=A_{P}$, a contradiction again. This concludes the proof.

3. An ideal $I$ with the property of $\operatorname{ann}(E)$ of (b) above, that is $\operatorname{ann}(I)=(0)$, is called dense. As it is well known, whether in general it contains a nonzero divisor depends on what the maximal rational extension of $A$ looks like. We consider here an example of an ideal in a local ring, with projective dimension one, but in which every element is a zero divisor.

Let $R=k[[x, y]]=$ power series ring in $x, y$ over the field $k$. Let $M$ be the $R$-module $=\bigoplus \sum k(P)$, where $k(P)=$ field of quotients of $R / P, P=$ prime ideal of height 1 . Let $A=R \oplus M$, where addition is componentwise and multiplication given by the rule

$$
(r, m) \cdot(s, n)=(r s, r n+s m) .
$$

Notice that $M$ is an ideal of $A, M^{2}=(0)$ and that $A$ is a local ring with maximal ideal $P$ generated by $x, y$. Observe also that every element of $P$ is a zero divisor. We claim that proj $\operatorname{dim} P=1$. For this end, consider $A^{2} \rightarrow P \rightarrow 0$ with $(1,0) \rightarrow x$ and $(0,1) \rightarrow y$. Let us determine the module of relations $L$ of $P$. Let $(a, b) \in L$; write $a=a_{0}+$ 
$\sum a_{P}$ (and similarly for $b$ ) where $a_{0}$ denotes the $R$-component of $a$ and $a_{P}$ its $k(P)$-component. Then

$$
a_{0} x+b_{0} y=0 \quad \text { and } \quad a_{P} x+b_{P} y=0 \quad \forall P .
$$

Since $x, y$ is a regular sequence in $R$, the first relation says that $\left(a_{0}, b_{0}\right)$ is a unique $R$-multiple of $(y,-x)$. As for the other relations, since $x, y$ cannot be both zero in $k(P)$ and this last is a field, $\left(a_{P}, b_{P}\right)$ must be a unique $k(P)$-multiple of $(y,-x)$. Thus $L=A(y,-x)$ and proj $\operatorname{dim} P=1$.

4. Corollary. Let $A$ be a commutative ring and let

$$
0 \rightarrow E_{n} \rightarrow \cdots \rightarrow E_{1} \rightarrow E_{0} \rightarrow E \rightarrow 0
$$

be a projective resolution of $E$ where the $E_{i}$ 's are finitely generated. Then ann $(\operatorname{ann}(E))$ is generated by an idempotent element.

Proof. For each prime ideal $P$, let $r(P)=\chi\left(E_{P}\right)$; since, for each $i, r k\left(\left(E_{i}\right)_{P}\right)$ defines a continuous function from Spec $A \rightarrow\{\boldsymbol{Z}+$ discrete topology $\}, r(P)$ is also a continuous function. Let $F(E)$ be the 0th Fitting invariant of $E$ and write $J=\operatorname{ann}(F(E))$; then, for each prime $P, J_{P}=\operatorname{ann}\left(F\left(E_{P}\right)\right)$ and is, by the Theorem, either $A_{P}$ or (0) -depending on whether $r(P)>0$ - or $=0$. This says that $A / J$ is a flat $A$-module (cyclic) with support an open set of Spec $A$. According to $[3$, p. 506] $A / J$ is $A$-projective and so $J$ is generated by an idempotent. It is clear that $J=\operatorname{ann}(\operatorname{ann}(E))$.

\section{REFERENCES}

1. M. Auslander and D. A. Buchsbaum, Codimension and multiplicity, Ann. of Math. (2) 68 (1958), 625-657. MR $20 \# 6414$.

2. I. Kaplansky, Commutative rings, Allyn and Bacon, Boston, Mass., 1970. MR $40 \# 7234$.

3. W. V. Vasconcelos, On finitely generated fat modules, Trans. Amer. Math. Soc. 138 (1969), 505-512. MR 39 \#199.

Rutgers University, New Brunswick, New Jersey 08903 